\title{
ON CUSPIDAL REPRESENTATIONS OF $p$-ADIC REDUCTIVE GROUPS
}

\author{
BY PAUL GERARDIN ${ }^{1}$
}

\author{
Communicated by Robert Fossum, February 12, 1975
}

\begin{abstract}
Let $k$ be a $p$-adic field, and $G$ a reductive connected algebraic group over $k$. Fix a maximal torus $T$ of $G$ which splits in an unramified extension of $k$, and which has the same split rank as the center of $G$. For each character $\theta$ of $T(k)$, satisfying some conditions, there is a cuspidal representation $\gamma_{\theta}$ of $G(k)$ which is a sum of a finite number of irreducible representations; the correspondence $\theta \mapsto \gamma_{\theta}$ is one-to-one on the orbits of such characters by the little Weyl group of $T$; furthermore, the formulas for the formal degree of $\gamma_{\theta}$ and its character for sufficiently regular elements of $T(k)$ are given: they are formally the same as is the discrete series for real reductive groups.
\end{abstract}

1. Unramified maximal tori. Let $k$ be a $p$-adic field, that is a finite extension of $\mathbf{Q}_{p}$ or a field of formal series over a finite extension of $\mathbf{F}_{p}$. We denote by $\bar{k}$ the residue field of order $q$.

Let $G$ be a reductive connected algebraic group defined over $k$, the derived group $G_{\mathrm{der}}$ of which is simply connected. A maximal torus of $G$ defined over $k$ is called minisotropic if it normalizes no (proper) horocyclic subgroup of $G$ defined over $k$.

Lemma. Suppose there exists a minisotropic maximal torus $T$ of $G$ which splits in a finite unramified extension $L$ of $G$. Then the Galois group $\Gamma$ of $L$ over $k$ has a unique fixed point $v$ in the apartment of $T$ in the building of $G_{\mathrm{der}}(L)[2]$; moreover, the face of $v$ is minimal amongst the faces in this apartment which are invariant by $\Gamma$.

2. Characters. We conserve notations and hypotheses of $\S 1$ and the Lemma. Let $\theta$ be a continuous character of $T(k)$. For each $\lambda \in X^{v}(T)$, the lattice of rational one-parameter subgroups of $T$, we define a character $\theta_{\lambda}$ of $L^{x}$ by

AMS (MOS) subject classifications (1970). Primary 22E50, 20G25; Secondary $20 \mathrm{C} 15$.

Key words and phrases. Reductive p-adic groups, cuspidal representations.

${ }^{1}$ Supported in part by National Science Foundation grant MPS72-05055A02. Copyright $\odot$ 1975, American Mathematical Society 


$$
\theta_{\lambda}(z)=\theta\left(\prod_{\Gamma}^{\gamma}\left(z^{\lambda}\right)\right), \quad z \in L^{x} .
$$

Definition 1. The character $\theta$ is called regular if, for every root $\alpha$ of $(G, T)$, the character $\theta_{\alpha^{v}}$ of $L^{x}$ associated to the coroot $\alpha^{v}$ is nontrivial.

For each root $\alpha$, we denote by $|\alpha|_{\theta}$ the conductor of $\theta_{\alpha^{v}}$ and let $R_{f}$ be the set of roots of $(G, T)$ such that $|\alpha|_{\theta} \leqslant f$.

Definition 2. The character $\theta$ is called good if it is regular and if, for every $f$, the set $R_{f}$ is a convex set of roots.

We need the following form of Macdonald's conjecture (cf. [1, C-6.7]):

Let $\bar{S}$ be a reductive connected algebraic group over $\bar{k}, \bar{T}$ a minisotropic maximal torus of $\bar{S}$; fix a finite extension $\bar{L}$ of $\bar{k}$ which splits $\bar{T}$; let $\Gamma$ be its Galois group. A character $\bar{\theta}$ of $\bar{T}(\bar{k})$ is called regular if, for every root $\alpha$ of $(\bar{S}, \bar{T})$, the character $z \mapsto \bar{\theta}\left(\Pi_{\Gamma}^{\gamma}\left(z^{\alpha^{v}}\right)\right)$ of $\bar{L}^{x}$ is nontrivial; if $\bar{\theta}$ is a regular character of $\bar{T}(\bar{k})$, then there exists a unique class $\bar{\sigma}_{\bar{\theta}}$ of representations of $\bar{S}(\bar{k})$ such that, if $\mathrm{St}_{\bar{S}}$ denotes the Steinberg representation of $\bar{S}(\bar{k}), \mathrm{St}_{\bar{S}} \otimes$ $\bar{\sigma}_{\bar{\theta}}$ is the induced representation $\operatorname{Ind}(\bar{S}(\bar{k}), \bar{T}(\bar{k}), \bar{\theta})$. Moreover $\bar{\sigma}_{\bar{\theta}}$ is cuspidal, and the intertwining number of two such representations $\bar{\sigma}_{\bar{\theta}_{1}}$ and $\bar{\sigma}_{\bar{\theta}_{2}}$ is equal to the number of elements in the little Weyl group of $\bar{T}$ in $\bar{G}(\bar{k})$ which send $\bar{\theta}_{1}$ on $\bar{\theta}_{2}$.

\section{Cuspidal representations.}

THEOREM. Let $G$ be a reductive connected algebraic group over the p-adic field $k$, the derived group $G_{\mathrm{der}}$ of which is simply connected. Let $T$ be a minisotropic maximal torus which splits in an unramified extension of $k$. Fix a good character $\theta$ of $T(k)$. Assume Macdonalds's conjecture and one of the following conditions:

(i) $|\alpha|_{\theta}>1$ for every root $\alpha$ of $(G(L), T(L))$

(ii) the residual characteristic of $k$ is not 2 and there exists a rational representation $\rho$ of $G_{\mathrm{der}}$ such that the corresponding bilinear form $\operatorname{Tr} \rho(X) \rho(Y)$ on the Lie algebra Lie $\bar{T}_{\mathrm{der}}(\vec{k})$ is nondegenerate.

Then there exists a class $\gamma_{\theta}$ of representations of $G(k)$ such that:

(a) $\gamma_{\theta}$ is a finite sum of irreducible representations of $G(k)$, the coefficients of which have compact support modulo the center;

(b) the intertwining number of two such representations $\gamma_{\theta_{1}}$ and $\gamma_{\theta_{2}}$ is the number of elements in the little Weyl group $W(T)$ of $T$ in $G(k)$ which send $\theta_{1}$ on $\theta_{2}$; 
(c) there exists a Haar measure on $G(k)$, independent of $\theta$, such that the formal degree of $\gamma_{\theta}$ is

$$
d(\theta)=\left(\prod_{R} q^{|\alpha|_{\theta}-1}\right)^{1 / 2}
$$

where $R$ is the set of roots of $(G, T)$;

(d) for $t \in T(k)$ such that $\operatorname{val}\left(t^{\alpha}-1\right) \geqslant|\alpha|_{\theta} / 3$ for every $\alpha \in R$, the value on $t$ of the character of $\gamma_{\theta}$ is given by

$$
\operatorname{Tr} \gamma_{\theta}(t)=(-1)^{l(G)}(-1)^{\Sigma_{R / \Gamma}\left(|\alpha|_{\theta}-1\right)} \sum_{W(T)} \frac{\theta}{\Delta}\left({ }^{w} t\right)
$$

where $l(G)$ is the split semisimple rank of $G, \Delta$ is the $W(T)$-invariant function on the regular elements of $T(k)$ given by

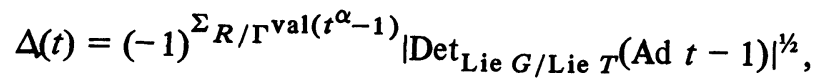

and $\Gamma$ is the Galois group over $\bar{k}$ of an unramified extension which splits $T$.

4. Remarks. 1. The proof is based upon an explicit construction of $\gamma_{\theta}$ (assuming Macdonald's conjecture), obtained by inducing a finite dimensional representation of a compact open subgroup of $G(k)$ naturally associated to $T$ and $\theta$; the essential tool is given by Weil's paper about Heisenberg groups [5]; we used too an argument given by $R$. Howe [4].

2. In the case where $|\alpha|_{\theta}$ is constant and strictly greater than 1 , and if the point $v$ of the lemma is special, the proofs are given in [3].

3. G. Lusztig has just proved Macdonald's conjecture.

\section{REFERENCES}

1. A. Borel, et al., Seminar on algebraic groups and related finite groups (The Institute for Advanced Study, Princeton, N. J., 1968/69), Lecture Notes in Math., vol. 131, Springer-Verlag, Berlin and New York, 1970. MR 41 \#3486.

2. F. Bruhat and J. Tits, Groupes réductifs sur un corps local. I, Inst. Hautes Études Sci. Publ. Math. No. 41 (1972), 5-262.

3. P. Gérardin, Sur les séries discrètes nonramifièes des groupes réductifs déployés p-adiques, Thèse, Paris, 1974; Lectures Notes in Math., Springer-Verlag, Berlin and New York (to appear).

4. R. Howe, Tamely ramified supercuspidal representations of $G L_{n}$ (preprint).

5. A. Weil, Sur certains groupes d'opérateurs unitaires, Acta Math. 111 (1964), 143-211. MR 29 \#2324.

SCHOOL OF MATHEMATICS, INSTITUTE FOR ADVANCED STUDY, PRINCETON, NEW JERSEY 08540 